\title{
Prevalencia de disfunción tiroidea en pacientes tratados con amiodarona en un hospital de la Ciudad de México
}

\section{Prevalence of thyroid dysfunction in patients treated with amiodarone in a hospital in Mexico City}

\author{
Jesús CAJIGAS-Silva* Y CARLOS T. VeGA-NAVA
}

Departamento de Medicina Interna, Hospital de Especialidades de la Ciudad de México "Dr. Belisario Domínguez", Secretaría de Salud, Ciudad de México, México

\section{RESUMEN}

La amiodarona ejerce múltiples efectos sobre la glándula tiroides para su correcto funcionamiento, se distingue al hipotiroidismo y a la tirotoxicosis asociada a amiodarona como las dos grandes categorías de disfunción tiroidea, caracterizadas por alteración en las pruebas de funcionamiento tiroideo. Se realizó un estudio observacional, descriptivo y longitudinal evaluando la función tiroidea de los pacientes tratados con amiodarona, obteniendo una prevalencia de disfunción tiroidea del $14.8 \%$, el $11.1 \%$ lo compone el hipotiroidismo y el $3.7 \%$ corresponde a tirotoxicosis asociada a amiodarona. No se observó relación entre la dosis semanal de amiodarona y la alteración tiroidea. Se obtiene un panorama general de la distribución y frecuencia de los diferentes tipos de alteración tiroidea en pacientes tratados con amiodarona. Además, se refuerza la idea de normalizar la estrecha vigilancia de la función de la glándula tiroides en pacientes tratados con amiodarona por cualquier patología cardiaca.

Palabras clave: Disfunción tiroidea. Hipotiroidismo. Tirotoxicosis. Amiodarona.

\section{ABSTRACT}

Amiodarone has multiple effects on the thyroid gland and its proper functioning. Hypothyroidism and thyrotoxicosis associated with amiodarone are distinguished as the two main categories of thyroid dysfunction, characterized by impaired thyroid function tests. An observational, descriptive and longitudinal study was carried out evaluating the thyroid function of patients treated with amiodarone, obtaining a prevalence of thyroid dysfunction of $14.8 \%, 11.1 \%$ corresponds to hypothyroidism and $3.7 \%$ corresponds to thyrotoxicosis associated with amiodarone. No relationship was observed between the weekly dose of amiodarone and thyroid alteration. An overview of the distribution and frequency of different types of thyroid abnormalities is obtained in patients treated with amiodarone, and the idea of normalizing close monitoring of thyroid gland function in patients treated with amiodarone for any cardiac pathology is also reinforced.

Key words: Thyroid dysfunction. Hypothyroidism. Thyrotoxicosis. Amiodarone.
Correspondencia:

*Jesús Cajigas-Silva

E-mail: jescasil@live.com.mx
Fecha de recepción: 02-09-2020

Fecha de aceptación: 27-10-2020

DOI: 10.24875/RME.20000089
Disponible en internet: 10-08-2021 Rev Mex Endocrinol Metab Nutr. 2021;8:120-5

2462-4144 / ( 2020 Sociedad Mexicana de Nutricion y Endocrinologia, AC. Publicado por Permanyer. Este es un artículo open access bajo la licencia CC BY-NC-ND (http://creativecommons.org/licenses/by-nc-nd/4.0/). 


\section{INTRODUCCIÓN}

La estrecha relación entre las hormonas tiroideas y la fisiología cardiovascular hace que los efectos de la amiodarona sobre la función tiroidea sean una consideración clínica importante cuando se monitoriza a pacientes con diversas formas de patología cardiaca que ameriten tratamiento con dicho antiarrítmico.

La prevalencia de disfunción tiroidea varía según la región, encontrando alteración tiroidea del 12$30 \%$ en pacientes tratados con amiodarona. La mayoría de los pacientes permanecerán eutiroideos durante todo el ciclo de tratamiento y entre el 10 al 20\% de los pacientes manifestarán hipotiroidismo asociado a amiodarona (HAA). El HAA es más frecuente en áreas con suficiente yodo, con un índice de incidencia para mujeres y hombres de $1.5: 1.2^{1-3}$.

La tirotoxicosis asociada a amiodarona (TAA) ocurre en el 2 al 10\% de los pacientes que reciben amiodarona, el riesgo predicho para TAA difiere entre las diferentes poblaciones y puede variar del $5-47 \%$ dependiendo de la presencia de factores clínicos adicionales. A diferencia del HAA, la TAA es más frecuente en hombres, con una relación hombre:mujer de 3:1. Como regla general, la TAA es relativamente más frecuente en áreas con deficiencia de yodo y el HAA en áreas con suficiente yodo ${ }^{4,5}$. En el 2010, en un estudio realizado en el Hospital Universitario de Valdecilla, España, se analizaron las pruebas de función tiroidea de 627 pacientes tratados con amiodarona, 155 de ellos tuvieron algún tipo de alteración tiroidea (24.7\%), la alteración más frecuente fue el hipotiroidismo $(9.7 \%)$, seguido de la tirotoxicosis $(8.1 \%)$ y 31 casos de disfunción en su forma subclínica $(4.9 \%)^{6}$.

\section{AMIODARONA}

La amiodarona es un agente antiarrítmico de clase III, un derivado yodado del benzofurano con estructura similar a las hormonas tiroideas, triyodotironina (T3) y tiroxina (T4), que contiene una gran cantidad de yodo; debido a estas características, el uso de amiodarona puede generar alteración en la glándula tiroides e inducir hipotiroidismo con elevación de la hormona estimulante de la tiroides (TSH) o tirotoxicosis con un nivel principalmente aumentado de $\mathrm{T3}^{7-10}$.

\section{Efectos de la amiodarona sobre la glándula tiroides}

Se recomienda realizar pruebas seriadas de la función tiroidea a las 8 semanas de iniciado el tratamiento, cada 3 meses durante el primer año y de 1-2 veces al año a partir del segundo año.

El HAA se genera por inhibición de la oxidación del yoduro (proceso indispensable durante la síntesis de hormonas tiroideas) debido al exceso de yodo intratiroideo, efecto conocido como Wolff-Chaikoff. La TAA surge esencialmente por una falla de los mecanismos autorreguladores de la tiroides que rigen su autonomía (efecto Jod-Basedow). Este efecto ocurre típicamente en áreas deficientes de yodo y en pacientes con enfermedades subyacentes como trastornos tiroideos nodulares o autoinmunitarios ${ }^{11-13}$.

Los efectos de la amiodarona y los efectos relacionados con el yodo alterarán la actividad de las enzimas deiodinasas. La inhibición de la deiodinasa 1 (D1) en los tejidos periféricos disminuye la concentración de T3, un aumento de la concentración total de T4 y un aumento de la concentración de T3 reversa. Además de la inhibición de la actividad de la D1 y la D2 en la hipófisis, la disminución del transporte de T4 intracelular y la disminución de la unión del receptor T3 a nivel hipofisiario conducen a un aumento en la concentración de $\mathrm{TSH}^{14-16}$.

\section{Tirotoxicosis asociada a amiodarona}

Hay dos tipos de TAA, la diferenciación puede ser difícil debido al hecho de que muchos pacientes exhiben tipos mixtos. La TAA tipo 1 comparte 
similitudes con el hipertiroidismo inducido por yodo y generalmente ocurre en pacientes que residen en lugares con deficiencia de yodo, por lo regular estos pacientes tienen disfunción tiroidea conocida o previamente no diagnosticada. La TAA tipo 2 generalmente ocurre en las glándulas tiroideas normales y es debida al resultado de la destrucción del tejido tiroideo causado por la tiroiditis inducida por las concentraciones elevadas de yodo ${ }^{17,18}$.

\section{TRATAMIENTO DE LA TIROTOXICOSIS ASOCIADA A} AMIODARONA

Desde un punto de vista general, todos los pacientes con TAA deben considerarse potencialmente en riesgo y requieren tratamiento de emergencia. La tiroidectomía total es actualmente la mejor opción para una restauración rápida del eutiroidismo. La tiroidectomía de rescate debe considerarse en pacientes con deterioro de la función cardiaca, pacientes con una enfermedad cardiaca subyacente grave y/o pacientes con arritmias malignas ${ }^{19}$.

\section{Hipotiroidismo asociado a amiodarona}

El HAA en los pacientes tratados con amiodarona se presenta como hipotiroidismo franco o en su forma subclínica. Aunque el HAA puede ocurrir en pacientes con una glándula tiroides aparentemente normal, con mayor frecuencia se desarrolla en pacientes con tiroiditis autoinmune crónica subyacente, con una mayor prevalencia en mujeres y en áreas sin deficiencia de yodo 20,21 .

\section{TRATAMIENTO DEL HIPOTIROIDISMO ASOCIADO A AMIODARONA}

El HAA se trata con levotiroxina y no hay necesidad de suspender la amiodarona si se considera esencial para la enfermedad cardiaca subyacente. El tratamiento del hipotiroidismo subclínico puede ser innecesario en algunos casos, particularmente en los ancianos, en vista del aumento potencial en el riesgo de eventos cardiovasculares. La función tiroidea se debe evaluar periódicamente, ya que existe el riesgo de progresión a hipotiroidismo manifiesto ${ }^{22}$.

\section{PLANTEAMIENTO DEL PROBLEMA}

La estrecha relación entre las hormonas tiroideas y los efectos de la amiodarona sobre el funcionamiento de la glándula tiroides son de suma importancia cuando se monitoriza a pacientes con diversas formas de enfermedad cardiaca. Por una parte, no hay seguimiento ni evaluación sistemática de las pruebas de función tiroidea en pacientes a quienes se les prescribe tratamiento con dicho antiarrítmico y además no se cuenta con datos de la prevalencia en México.

OBJETIVO

Identificar la prevalencia de disfunción tiroidea evidenciada por alteración en las pruebas de funcionamiento tiroideo en pacientes tratados con amiodarona.

\section{MATERIAL Y MÉTODOS}

\section{Diseño del estudio}

Estudio observacional, descriptivo, longitudinal y prospectivo.

\section{Universo del estudio}

Todos los pacientes de la consulta externa y hospitalización de Medicina Interna del Hospital de Especialidades de la Ciudad de México "Dr. Belisario Domínguez" que cumplieran con los criterios de inclusión de este estudio durante el periodo comprendido del 1 de noviembre del 2019 al 31 de marzo del 2020. 


\section{Criterios de Inclusión}

Sexo indistinto, edad igual o mayor a 18 años y que recibiesen tratamiento con amiodarona.

\section{Criterios de no inclusión}

Patología tiroidea preexistente, tratamiento con amiodarona en un lapso menor de ocho semanas, pacientes con sustitución tiroidea o en tratamiento con fármacos antitiroideos.

\section{Plan de análisis estadístico}

Estadística descriptiva: para el análisis de las variables cualitativas se estimaron frecuencias absolutas y relativas mediante el porcentaje y frecuencia; para las variables cuantitativas se estimaron medidas de tendencia central y de dispersión.

\section{Consideraciones éticas y de bioseguridad}

- El estudio fue evaluado y dictaminado por el Comité de Ética en Investigación del Hospital de Especialidades "Dr. Belisario Domínguez".

- Se incluyó un proceso de consentimiento informado para solicitar la autorización de participar en el estudio.

\section{RESULTADOS}

Se obtuvo una muestra de 27 pacientes, de los cuales 14 fueron de sexo masculino y 13 de sexo femenino; de acuerdo con el grupo etario se conformó por 12 pacientes de 61-80 años, 11 pacientes en el grupo de 41-60 años y 4 pacientes de más de 81 años.

La dosis semanal de amiodarona en miligramos se agrupó en cuatro rangos, 13 pacientes con una dosis mayor a 1,300 mg, teniendo después a los grupos de $701-1,000 \mathrm{mg}$ y $1,001-1,300 \mathrm{mg}$ con la misma frecuencia, 6 pacientes en cada uno y el cuarto grupo integrado por 2 pacientes que reciben de 400-700 mg.
Disfunción tiroidea asociada a amiodarona en la población estudiada

Al comparar las pruebas de funcionamiento tiroideo basales y las obtenidas ocho semanas posteriores al inició del tratamiento con amiodarona se encontró que 23 pacientes no presentaron disfunción y en 4 pacientes se identificó algún tipo de disfunción (14.8\%), 3 de ellos catalogados como HAA y 1 con TAA.

\section{Hipotiroidismo asociado a amiodarona}

Al analizar los casos de HAA se distingue en mayor proporción el hipotiroidismo subclínico, conformado por dos pacientes y a un paciente con hipotiroidismo manifiesto.

\section{Tirotoxicosis asociada a amiodarona}

Dentro de la disfunción tiroidea de tipo tirotoxicosis se detectó a un paciente, mismo al que se le realizó ultrasonido tiroideo, estudio reportado sin alteraciones y por tanto categorizado como TAA tipo 2. No se encontró ningún caso de tirotoxicosis tipo 1 ni tirotoxicosis mixta.

\section{Tipo de disfunción tiroidea y dosis semanal de amiodarona}

De los casos de hipotiroidismo, ningún paciente se identificó en el grupo de 400-700 mg de amiodarona semanales, un paciente en el grupo de 701-1000 $\mathrm{mg}$, un paciente en el grupo de 1,001-1,300 mg y un paciente en el grupo de más de 1,300 mg. El caso de tirotoxicosis pertenece al grupo que recibe más de 1,300 mg de amiodarona de forma semanal.

\section{DISCUSIÓN}

Las alteraciones tiroideas tempranas asociadas al uso de amiodarona se presentan dentro de las ocho semanas posteriores al inicio del tratamiento con el antiarrítmico, no existe una dosis específica que se relacione con alteración en las pruebas de funcionamiento 
tiroideo. Se distinguen dos tipos principales de alteración, el HAA, mismo que se subdivide en hipotiroidismo manifiesto e hipotiroidismo subclínico; la otra forma de disfunción es la tirotoxicosis, conociéndose tres subtipos de esta alteración: TAA tipo 1, usualmente vista en glándulas tiroideas con afección o alteración estructural preexistente, tal es el caso del bocio multinodular, y tirotoxicosis tipo 2 , presente en glándulas tiroideas sin alteración, y finalmente los casos de tirotoxicosis mixta, que comparten similitudes de la forma 1 y 2 .

En el periodo comprendido de enero del 2002 a diciembre del año 2003 en el Hospital Universitario Marqués de Valdecilla se estudiaron 1,626 pacientes tratados con amiodarona, el $61 \%$ de ellos no se realizó ningún control de pruebas tiroideas, quedando por tanto excluidos del estudio; de los 627 restantes, 155 tuvieron algún tipo de disfunción (24.7\%) objetivada por alteración en las pruebas de funcionamiento tiroideo. La alteración tiroidea más frecuente fue el hipotiroidismo (9.7\%), seguido del hipertiroidismo (8.1\%) y hubo 31 disfunciones tiroideas subclínicas (4.9\%). En la población de nuestro hospital se identificaron 3 pacientes con alteración tiroidea, teniendo así una prevalencia del $14.8 \%$, 2 pacientes con hipotiroidismo ( 1 paciente con hipotiroidismo franco y 1 paciente en la forma subclínica) y 1 con tirotoxicosis. La discrepancia entre los resultados encontrados en nuestro estudio y el antes citado se podría atribuir a la diferencia entre las poblaciones (española y mexicana) y a la disimilitud en las concentraciones de yodo en los suelos de las diferentes regiones. Es importante resaltar que el tamaño de muestra también difiere, siendo una proporción mayor en el estudio de Valdecilla, encontrando un 59.9\% más de casos con algún tipo de alteración tiroidea.

En el presente estudio no se identificó una tendencia clara entre la dosis semanal de amiodarona y el desarrollo de alguno de los diferentes tipos de disfunción tiroidea.

\section{CONCLUSIONES}

Es de suma importancia el monitoreo de los pacientes tratados con amiodarona, son bien conocidas las vías patogénicas que conducen a la disfunción de la glándula tiroides por efectos de dicho antiarrítmico, sin embargo, y a pesar de tal aseveración, no se cuenta con protocolos concretos que establezcan la necesidad del uso sistemático de pruebas de funcionamiento tiroideo.

Con este estudio se obtiene un panorama general de la distribución y prevalencia de los diferentes tipos de alteración tiroidea en pacientes que reciben amiodarona, además se refuerza la idea de la necesidad del desarrollo, estandarización y aplicación de protocolos bien establecidos que normen conducta para la evaluación tiroidea con finalidad de ofrecer tratamiento y seguimiento oportuno.

\section{AGRADECIMIENTOS}

Agradecemos al Departamento de Medicina Interna, a todo el personal médico y de enfermería y al Laboratorio Clínico del Hospital Belisario Domínguez.

FINANCIAMIENTO

Financiamiento interno.

\section{CONFLICTO DE INTERESES}

Los autores declaran no tener conflicto de intereses.

\section{RESPONSABILIDADES ÉTICAS}

Protección de personas y animales. Los autores declaran que para esta investigación no se han realizado experimentos en seres humanos ni en animales.

Confidencialidad de los datos. Los autores declaran que han seguido los protocolos de su centro de trabajo sobre la publicación de datos de pacientes. 


\section{Derecho a la privacidad y consentimiento infor- mado. Los autores declaran que en este artículo no aparecen datos de pacientes.}

\section{BIBLIOGRAFÍA}

1. Cohen J, Dahl P, Danzi S, Klein I. Effects of amiodarone therapy on thyroid function. Nat Rev Endocrinol. 2010;6:34-41.

2. Soto JR, Verbeke SM. Disfunción tiroidea y corazón. Med Clin Condes. 2015;26(2):186-97.

3. Benjamens S, Dullaart R, Slulter WJ, Rlenstra I, LinksT. The clinical value of regular thyroid function tests during amiodarone treatment. Eur J Endocrinol. 2017;177:9-14.

4. Benvenga S, Tuccari G, Leni A, Vita R. Thyroid gland: Anatomy and physiology. En: Huhtaniemi I, Martini L, editores. Encyclopedia of Endocrine Diseases (Second Edition). Vol. 4. Elsevier; 2018. pp. 382-90.

5. Taylor PN, Albrecht D, Scholz A, Buey G, Lazarus JH, Colin MD. Global epidemiology of hyperthyroidism and hypothyroidism. Nat Rev Endocrinol. 2018;2014:301-16.

6. Casuso E, Nan D, Hernández JL, García O, Alonso J, González J. Disfunción tiroidea inducida por amiodarona. Análisis en condiciones de práctica clínica habitual. Med Int Mex. 2011;27(3):231-7.

7. Hofman A, Nawara C, Ofluoglu S, Holzmannhofer J, Strohmer CP. Incidence and predictability of amiodarone-induced thyrotoxicosis and hypothyroidism. Middle Euro J. 2008;120:493-8.

8. Hyoung SP, Yoon NK. Adverse effects of long-term amiodarone therapy. Korean J Intern Med. 2014:29:571-3.

9. Jabbar A, Pinigitore A, Pearce SH, Zaman G, Razvi S. Thyroid hormones and cardiovascular disease. Nat Rev Cardiol. 2017;14:39-51.
10. Ghosh R. Thyroid dysfunction in long term amiodarone therapy [Internet]. GM. Endocrinology-Midlife and Beyond; octubre de 2010. Disponible en: https://www.gmjournal.co.uk/media/21714/oct2010p547.pdf

11. Danzi S, Klein I. Amiodarone-induced thyroid dysfunction. J Intensive Care Med. 2015;30(4):179-85

12. Menhraein F. Review on amiodarone as an antiarrhythmic drug. En: Breijo-Marquez R, editor. Abnormal heart rhythms. IntechOpen; 2015.

13. UK Guidelines for the use of thyroid function tests [Internet]. The Association for Clinical Biochemistry, British Thyroid Association, British Thyroid Foundation; julio de 2006. Disponible en: https://www.britishthyroid-association.org/sandbox/bta2016/uk_guidelines_for_the_use_ of_thyroid_function_tests.pdf

14. Mullur R, Liu Y, Brent GA. Thyroid hormone regulation of metabolism Physiol Rev. 2013;94:355-82.

15. Fernández LR, López A, Santisteban P. Thyroid transcription factors in development, differentiation and disease. Nat Rev Endocrinol. 2015;11(1):29-42.

16. Wolmarans DW. Maintaining euthyroidism: Fundamentals of thyroid hormone physiology, iodine metabolism and hypothyroidism. South Afric Pract. 2017:59(4):11-21.

17. Meytal A, Tsadok P, Jackevicius MS, Elham R, Vidal E, Eisenberg MD Amiodarone-induced thyroid dysfunction: brand-name versus generic formulations. Canadian Med Association. 2011;12:17-23.

18. Ahmed S, van Gelder IC, Wiesfeld AC, van Veldhuisen DJ, Links TP. Determinants and outcome of amiodarone-associated thyroid dysfunction. Clin Endocrinol (Oxf). 2011;75(3):388-94

19. Fuks AG, Vaisman M, Buescu A. Thyroid dysfunction and cardiological management in patients receiving amiodarone. Arquivos Brasileiros Cardiol. 2004;82:1-5.

20. Zimmermann. MB, Boelaert K. lodine deficiency and thyroid disorders. Lancet Endocrinol. 2015;14:1-10.

21. Lyle A, Sidoway MD. Amiodarone: Guidelines for use and monitoring. Clinical Pharmacol. 2003;68(11):2189-96.

21. Bartalena L, Bogazzi F, Chiovato L, Hubalewska D, Links T, Vanderpump M. European Thyroid Association (ETA) Guidelines for the Management of Amiodarone-Associated Thyroid Dysfunction. Eur Thyroid J. 2015;7:55-66. 